\title{
Smart Intersection Design for Traffic, Pedestrian and Emergency Transit Clearance using Fuzzy Inference System
}

\author{
Aditi Agrawal ${ }^{1}$, Rajeev Paulus ${ }^{2}$ \\ Department of Electronics and Communication Engineering \\ VIAET, SHUATS, Prayagraj, India
}

\begin{abstract}
Traffic flow is regulated and controlled with the aid of traffic signals implemented at all major intersections in urban areas. With the increase in vehicles, the traditional control strategies are incapable of clearing heavy traffic which leads to long traffic queues and prolonged waiting time at intersections. Smart cities are increasingly adopting solutions by developing smart traffic lights to improve the flow of vehicles. A major demand arises to increase the efficiency of traffic controllers with the objective to minimize traffic congestion, prioritize emergency transit and give way to pedestrians to cross the lanes at an intersection. This requires leveraging the existing techniques that identify the best solutions at the lowest possible cost. This paper proposes Fuzzy Adaptive Control System (FACS) that uses fuzzy logic to decide the phase sequence and green-time for each lane based on sensed input parameters. It is designed with an aim to improve traffic clearance at isolated intersection especially in peak traffic hours of the day along with giving precedence to emergency vehicle as soon as it is detected and also assist pedestrian passage thus reducing their waiting time at the intersection. Performance of the proposed Fuzzy Adaptive Control System (FACS) is evaluated through simulations and compared with Pre-Timed Control System (PTCS) and Traffic Density-based Control System (TDCS) at a busy intersection with lanes leading to offices, schools and hospitals. Simulation results show significant improvement over PTCS and TDCS in terms of traffic clearance, immediate addressing of the emergency vehicle and giving preference to pedestrian passage at the intersection.
\end{abstract}

Keywords-Adaptive traffic light control; smart intersection; fuzzy logic; emergency vehicle; pedestrian crossing

\section{INTRODUCTION}

Traffic signals are installed at road intersections and play a major role to control the traffic flow and avoid congestion. The increased number of vehicles and greater urbanization create critical problems of traffic jam, increased wait time and fuel consumption at intersections. This problem occurs mainly due to the most common version of traffic light controllers, the Pre-Timed Control System (PTCS) that gives way to lanes based on fixed signalling plans. This system is easy to install but is effective only in situations with low traffic density that does not show large variations in time. In case of heavy traffic and varied flow, it is necessary to keep a greater control over the traffic configuration at intersections. Some intersections are equipped with Traffic Density-based Control System (TDCS) that uses information on current traffic obtained from detectors and necessary control logic to prioritize certain phases or traffic movements to be serviced. This system shows improved performance over PTCS. However, they are ineffective in clearing heavy traffic during peak hours. An effective approach is an adaptive and intelligent design that can respond to the random traffic flow behavior and even consider other decision parameters. Rule-based fuzzy logic control scheme helps in the development of multi-criteria control procedures very similar to human thinking and can best replace an ideal policeman at the intersection. Use of fuzzy logic in taking decisions for existence of roundabout to assist in path planning of a wheeled mobile robot is presented in [1]. In an in-depth review [2] the authors have summarized a wide literature of fuzzy logic-based traffic light controllers in an effective tabular representation. A detailed survey of use of in-vehicle and on-road sensors to serve as data collection components in Intelligent Transportation System (ITS) is discussed in [3]. Comparison between different sensor technologies giving the advantages and disadvantages was also discussed.

Fuzzy logic started by Zadeh [4] has been commonly used by researchers in solving traffic congestion problem at intersections and the development of fuzzy logic in traffic control was extensively discussed in [5]. The advantage of using this kind of traffic signal control in Saudi Arabia was illustrated in [6]. A fuzzy based system used to adjust the phase sequence and duration of traffic lights at isolated intersection [7] was tested by collecting real time data from signalized intersection in State of Kuwait. This system showed improved performance in case of heavy traffic volume. Various other research efforts have been conducted to combine fuzzy logic in providing solution for traffic management at intersections. An isolated T-junction was considered [8] and fuzzy logic-based traffic light controller was designed by taking fuzzy inputs as vehicles on arrival side, queue side and right side to decide for the output variable extension time. Better performance was achieved in terms of decreased waiting time but the authors proposed to conduct future research by taking pedestrians and emergency movement into account. A two-stage fuzzy control for traffic light was suggested [9] by calculating traffic urgency degree for all red phases using traffic urgency evaluation module and a decision module to decide green time extension of current green phase. Similar two stage fuzzy system [10] included the first stage named as urgency decision module which decided the next green phase based on urgency. The second module 
calculated the green time extension of the chosen phase with the help of queue length as input. Another work [11] considered the possibility to change the green light duration at an isolated four-lane intersection using fuzzy inference system by taking the road condition, traffic and time of the day as major deciding inputs. Researchers in [12] applied fuzzy logic to improve traffic light by taking queue length, arrival flow and exit flow as inputs and calculated the urgency degree using fuzzy rules. The variable cycle length was obtained by extending or shortening the phase time in accordance with the urgency degree. Comparison with fixed time control system showed significant improvement. Traffic flow of a four-way intersection and T-crossing was studied in [13] and traffic flow probability for the lanes was considered. The designed fuzzy logic controller used inputs as queue length and waiting time of vehicles, decided using the rule base for the output variable green-time. This traffic light system followed the fixed phase sequence and only altered the green time of the lanes. Results showed significant improvement over the static phase traffic light system. A recent research [14] used queue lengths as input given to two controllers used to select the green phase and decide the green time. These designs showed improved performance as compared to pre-timed system by reducing the average waiting time of vehicles but in the absence of emergency transit and pedestrian consideration.

In view of the risk of pedestrians who are waiting long to cross the lane while green and their proneness to accidents, the adaptive traffic light design must also consider giving way to pedestrians while addressing traffic at the intersection. Another important consideration is giving way to emergency transit such as ambulance, fire brigade, police van, etc. immediately as they are detected at the intersection. Some research works that considered emergency transit includes [15] by using three-stage fuzzy control. They considered queue length and waiting time of vehicles as two input variables and green time extension as output variable of the first stage. The output from the first stage works as input to the next stage and the third stage switches current phase to the demanded next phase by output of its previous stage. A separate function block was developed to detect emergency vehicle siren and switch to green to prioritize its passage which added to the design complexity. Fuzzy control system [16] designed by taking queue lengths, traffic arrival rate and emergency vehicle as inputs to two controllers for phase selection and green-time extension. Simulation results showed noticeable improvements when compared with pre-timed system. However, in real time scenario the use of two controllers may lead to high response time as compared to a single controller design. A dynamic traffic management center was proposed in [17] to determine the priority of road segment using fuzzy logic. The two input parameters taken namely vehicle count and presence of emergency vehicle were taken to output the phase priority. Green duration was calculated mathematically on account of the number of vehicles present on the road segment. The designed controller prioritized emergency vehicle clearance and optimized wait time of vehicles at intersection. This work also used congestion-aware routing algorithm to transmit sensed data from roadside sensors to the controller with minimum delay. The authors further proposed to test and validate the designed system in the real time scenario and also proposed to work on connected intersections.

A smart portable wireless control system for pedestrian crossing was developed [18] to manage the traffic automatically and assist the pedestrians to cross the road safely. The system infrastructure and cost- effective design finds application to develop smart pedestrian crossings especially near schools. Smart solution to regulate traffic lights in signalized pedestrian crossings by use of fuzzy logic controller was also proposed [19]. Time of the day and the number of pedestrians about to cross the road were considered. The pedestrian flow was analyzed and performance was compared with static traffic lights. The designed system reduced the average queue length of the pedestrians waiting to cross. A controller using three fuzzy modules [20] was designed to find the extension degree of green phase and urgency degree of red phases. The controller showed enhanced performance under low and medium traffic conditions but only small improvement was achieved under heavy traffic conditions. The past works projected the need of an integrated design to support vehicle, emergency and pedestrian clearance at the intersection.

This paper proposes an integrated design of Fuzzy Adaptive Control System (FACS) with an objective of reducing congestion, prioritizing emergency transit and giving way to pedestrians thus, reducing their waiting time at the intersection. Another feature of the designed system is that the design makes use of a single controller with four fuzzified inputs and two fuzzified outputs and an optimized rule base. This simple design helps in reducing the response time of the controller which is a desirable feature in real time applications. To evaluate the performance of proposed FACS a realistic traffic model is used to obtain traffic variation for 24 hours of the day at a busy intersection.

\section{PROPOSED SySTEM DESIGN}

\section{A. Implementation Scenario}

The traffic intersection under study is considered to be a four-lane intersection. This is assumed to be one of the major and most occupied intersection of an urban area with lanes leading to offices, schools and hospitals. So, a need arises incorporate an intelligent traffic light system to manage traffic, emergency transit along with giving way to pedestrians to cross. The real-time traffic inflow on the lanes existing throughout the day is modelled as a stochastic process. The implementation scenario of the proposed system at a congested intersection is shown in Fig. 1. The vehicle and pedestrian detectors are employed as considered in [20]. Roadside detectors are used to count vehicles on lanes and a push-to-walk button is deployed at sidewalks to collect information about pedestrians. The pedestrians who wish to cross the road can push this button and timer gives information about the duration since button is pressed. This duration is termed as Pedestrian Wait Time (PWT). Acoustic sensors are deployed way ahead of the intersection to sense the emergency vehicle headed towards the intersection. 


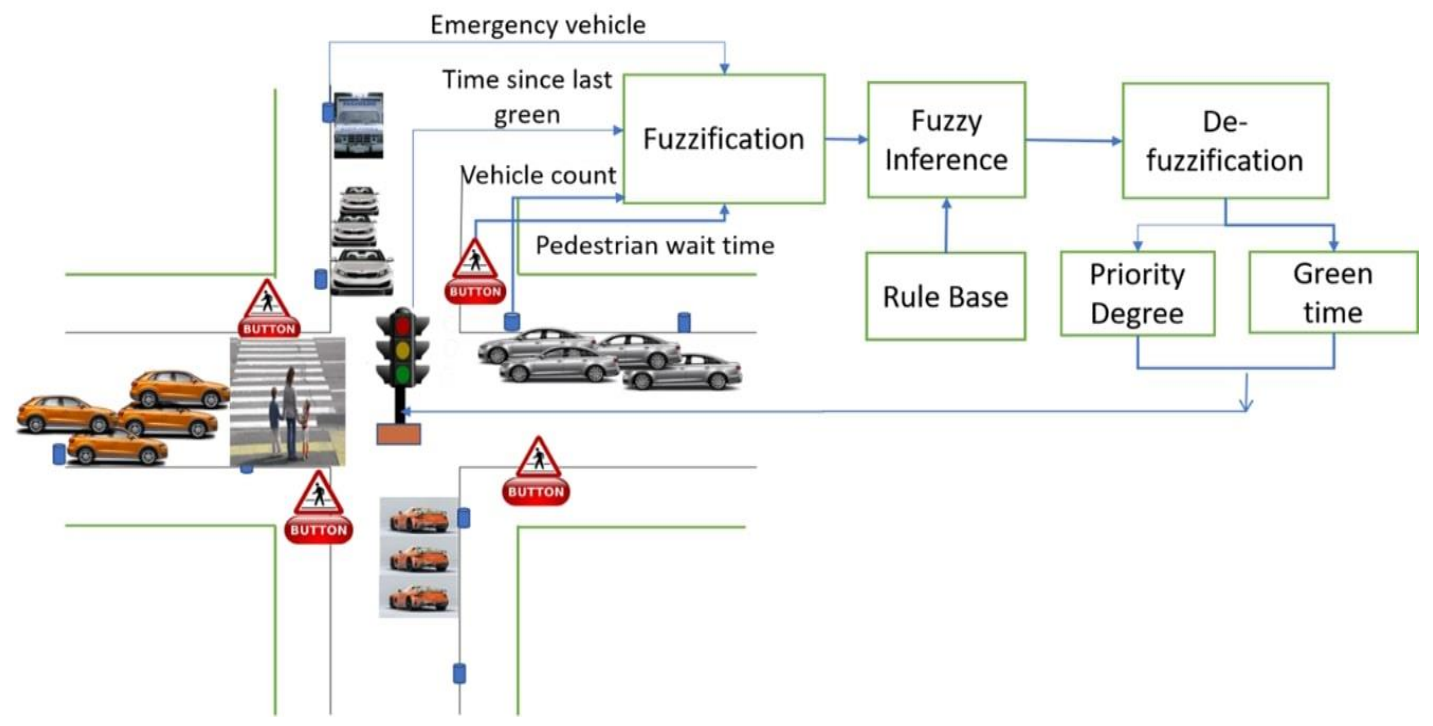

Fig. 1. Implementation Scenario of Proposed Fuzzy Adaptive Control System.

\section{B. Fuzzy Inference System Design}

The proposed FACS system is designed and modelled in MATLAB using Fuzzy Logic Toolbox.

A simplistic approach having four inputs and two outputs is used in order to design FACS. The inputs are taken as Vehicle Count (VC), Pedestrian Wait Time (PWT), Time since last Green (TG) and Emergency Transit (ET) as they are important parameters in deciding the green passage for lanes. Two outputs of the fuzzy inference system are Priority Degree (PD) and Green Time (GT). A single controller is preferred over multiple controllers in stages for low data processing and realistic control actuation times. Fuzzy Parameters and their membership function design are as shown in Fig. 2 to 7. Trapezoidal and triangular membership functions are used to represent the input and output fuzzy variables.

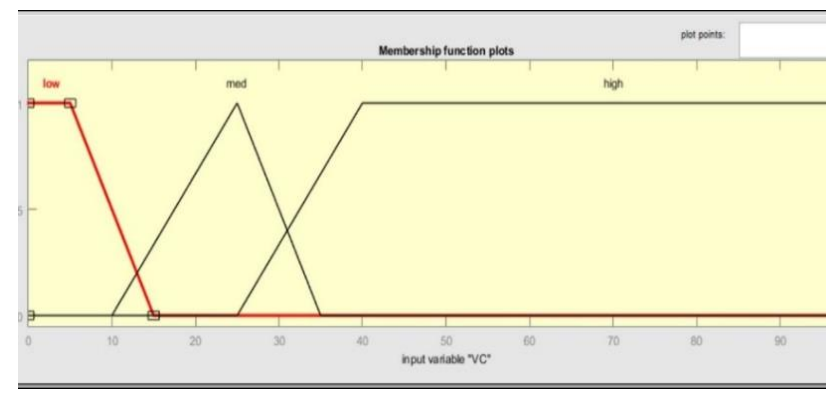

Fig. 2. Membership Function of Vehicle Count.

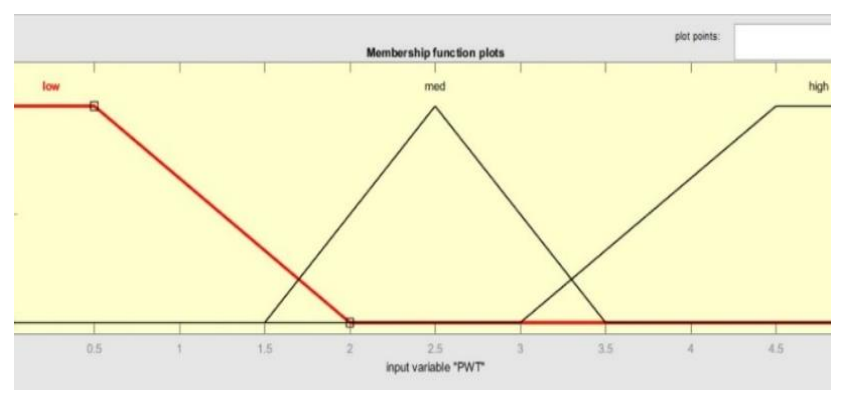

Fig. 3. Membership Function of Pedestrian Wait Time.

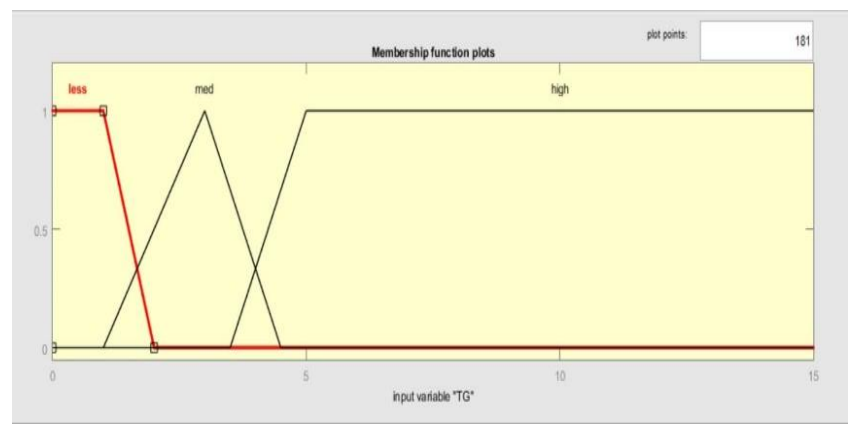

Fig. 4. Membership Function of Time since Last Green.

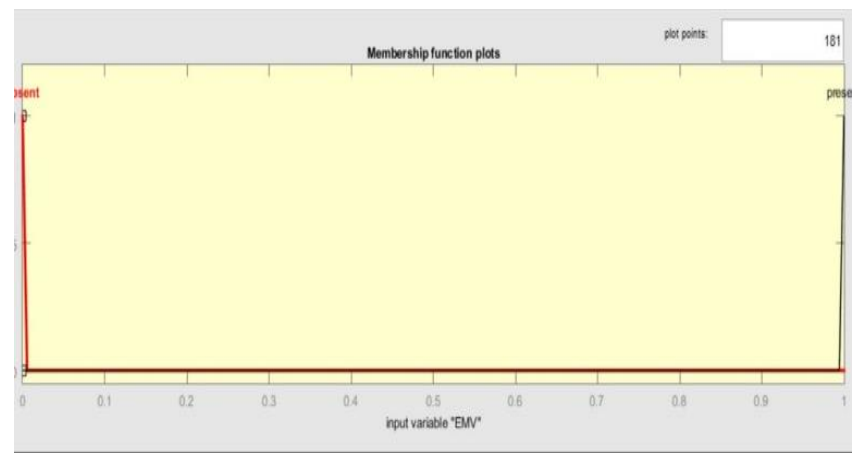

Fig. 5. Membership Function of Emergency Transit.

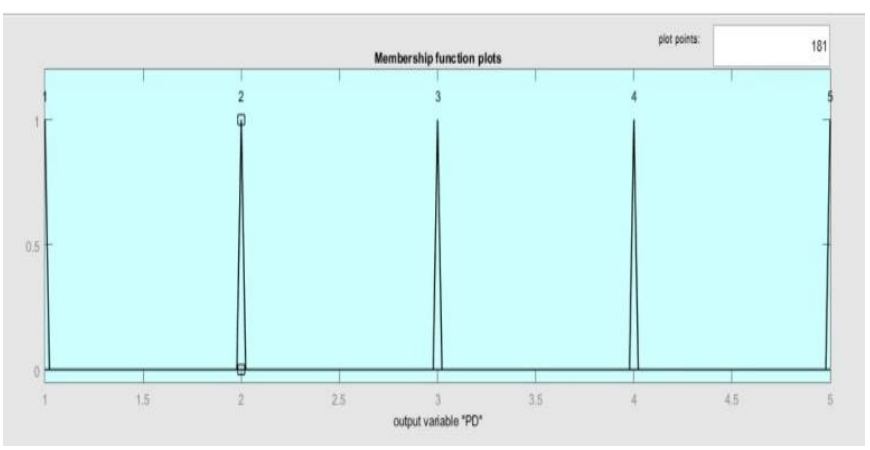

Fig. 6. Membership Function of Priority Degree. 




Fig. 7. Membership Function of Green Time.

\section{Rule Base Design}

Rules of the fuzzy inference system are fabricated with an aim to maximize traffic clearance even in peak traffic hours along with highest priority to emergency transit and preference to pedestrian waiting on the sidewalk to cross. While designing the rules in MATLAB's fuzzy inference system highest priority is given to the lane detected with Emergency Transit (ET). Vehicle Count (VC) is considered as the second most important factor as it indicates the degree of traffic congestion. The lane with maximum Pedestrian Wait Time (PWT) is given low priority for green phase so that pedestrians could cross. Time since last Green (TG) is an input to avoid the situation when lane with low traffic does not get green signal and vehicles in that lane undergo a prolonged waiting time. The output is Priority Degree (PD) for the lanes. It is arranged in decreasing order for green phase sequencing in next cycle. The second output is Green Time (GT) that corresponds to the time for which the traffic signal is green for the corresponding lane. The Rule editor of the proposed fuzzy system is given in Fig. 8. Design is optimized with 30 rules in the rule base. Less number of rules minimizes the processing time of the controller and hence fast response time is achieved which is a desirable QoS parameter while dealing with real time inputs.

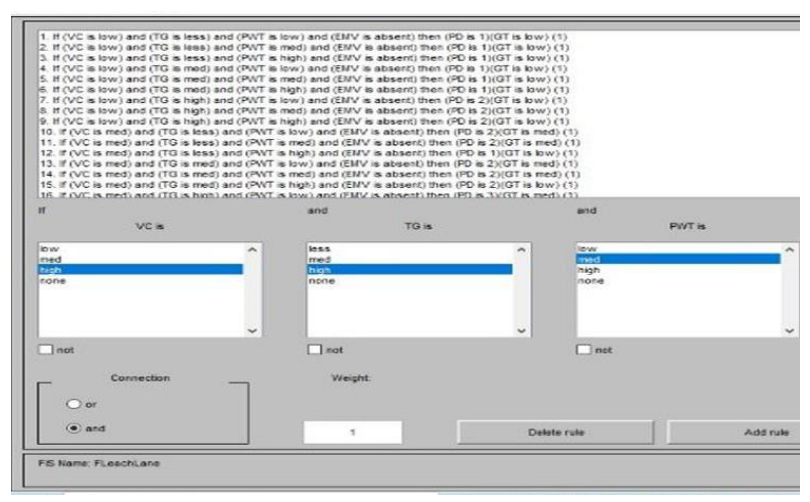

Fig. 8. Rule Editor of Proposed Fuzzy Adaptive Control System.

\section{3-D Surface Plots of Fuzzy Inference System}

After designing the rule base of proposed FACS, the impact of input variables on output variables can be seen by studying the surface plots. Surface plot in Fig. 9 illustrates that output variable Green Time (z-axis) rises and takes maximum value as the Vehicle Count (x-axis) increases at low values of Ped Wait Time (y-axis) whereas at high values of Ped Wait Time (PWT) the Green Time (GT) rises with increasing
Vehicle Count (VC) but takes low values to allow traffic to pass as well as ensuring that even pedestrians do not wait for increased time.

Surface plot in Fig. 10 shows that output variable Priority Degree (z-axis) has maximum value when Vehicle Count (yaxis) is high but decreases with increasing Ped Wait Time ( $\mathrm{x}$ axis). The value of Priority Degree (PD) decreases sharply if Ped Wait Time (PWT) is high for low value of Vehicle Count (VC).

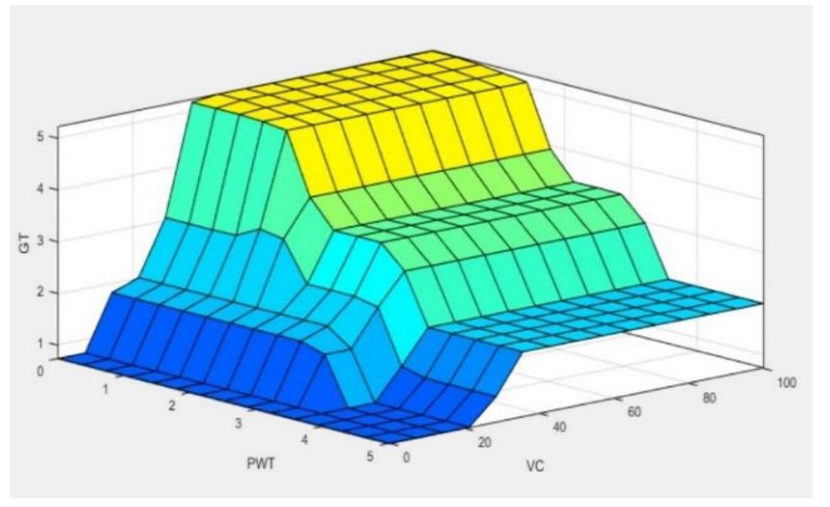

Fig. 9. Surface Plot of Output Variable Green Time.

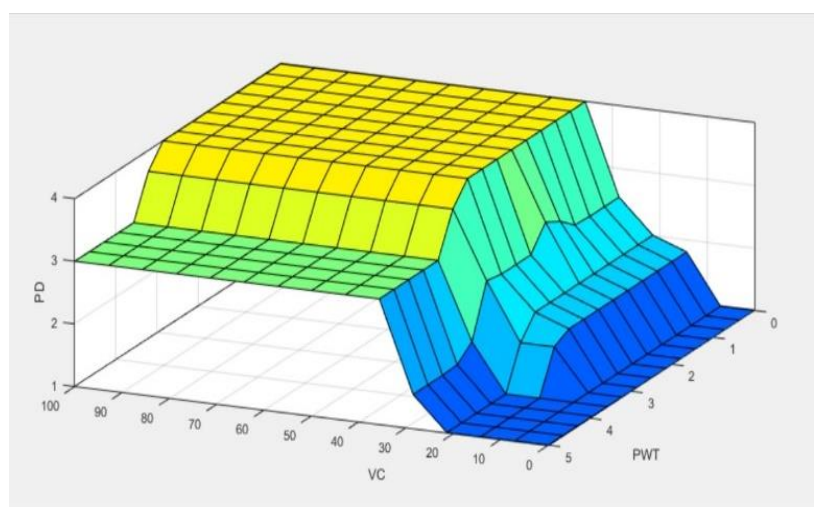

Fig. 10. Surface Plot of Output Variable Priority Degree.

\section{SIMULATION SETUP}

\section{A. Traffic Distribution}

Traffic analysis and good traffic modelling is an essential requirement for accurate planning of traffic capacity. There are many traffic models among which the Poisson distribution [21] has been widely used to model the incoming traffic [8]. Number of vehicles arriving per unit of time interval has been modelled using parameter $\lambda$ of the Poisson distribution. A realistic simulation environment is chosen to model a busy traffic intersection. The traffic distribution on lanes at various intervals of time in the day has been characterized by values of $\lambda$ as stated in Table $\mathrm{I}$.

Random distribution of vehicle arrival at low traffic hours $(\lambda=8)$ and peak traffic hours $(\lambda=25)$ obtained for 100 iterations is shown in Fig. 12. The traffic flow on any lane for the entire day showing peak traffic hours to emulate actual traffic scenario at the intersection is represented in Fig. 11. The time of the day between 10 am to $11 \mathrm{am}$ and $4 \mathrm{pm}$ to $6 \mathrm{pm}$ are considered to be the peak traffic hours. 


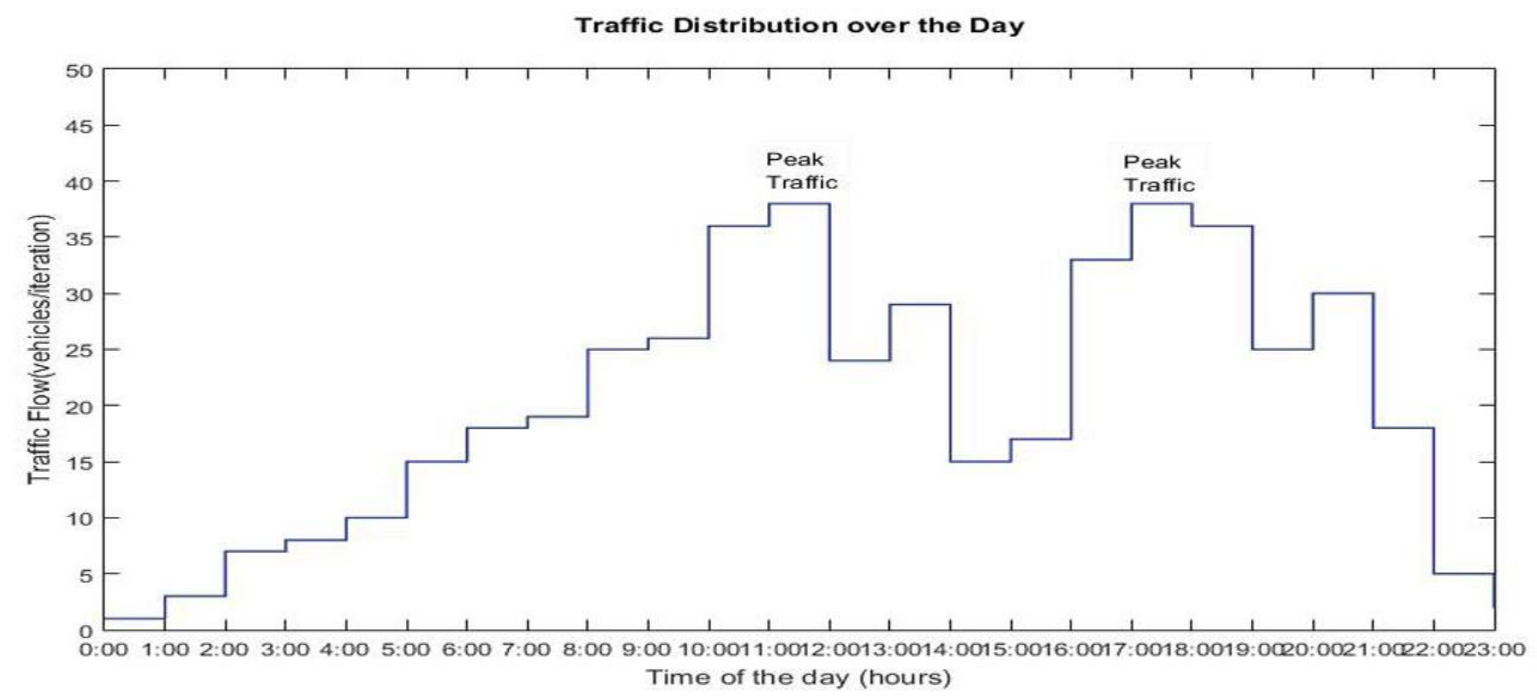

Fig. 11. Traffic Flow on a Lane throughout the Day Showing the Peak Traffic Hours.

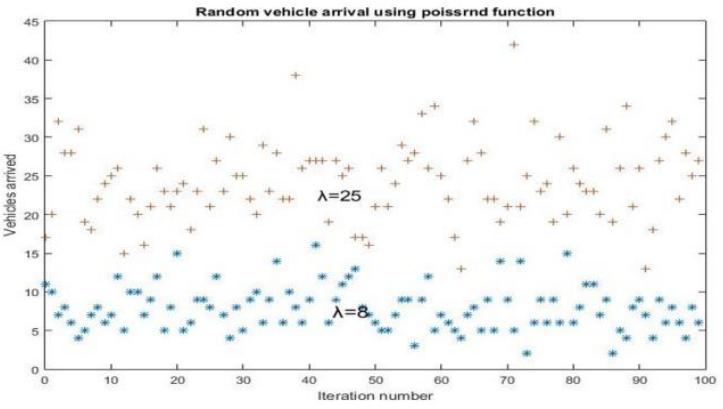

Fig. 12. Random Distribution of Vehicle Arrival.

\section{B. Comparative Analysis}

Performance of the proposed FACS is evaluated by comparing it with the PTCS and TDCS. The pre-timed control system (PTCS) is considered to have a regular green light switching sequence and fixed green time of one minute irrespective of the traffic conditions. This is the traditional system which is mostly implemented at intersections. The traffic density-based control system (TDCS) is designed to switch green light between lanes and adjust the green light time in pre-defined steps of half, one and one and a half minute according to the vehicle density at lanes. The proposed fuzzy adaptive control system (FACS) decides the lane sequence and green time by fuzzification of inputs and application of rules from the designed rule base. The output from the inference engine is de-fuzzified to generate priority degree and green time for lanes.

\section{Simulation Scenario}

The three systems PTCS, TDCS and FACS considered for performance evaluation are simulated under same traffic conditions for each hour of the day in ten observation sets of 100 iterations each. The traffic distribution on all the lanes of the intersection is identical and varies in accordance with the time of the day as in Table I. Emergency vehicles are assumed to enter any lane randomly and the probability of detecting an emergency vehicle is more during the peak traffic hours. A random pedestrian wait time varying from zero to maximum value of GT is generated for each lane.

TABLE I. TRAFFic DENSITY ON LANES FolLOWING POISSON DISTRIBUTION

\begin{tabular}{|l|l|l|}
\hline $\begin{array}{l}\text { Time of the day } \\
\text { (hours) }\end{array}$ & Value of $\lambda$ & $\begin{array}{l}\text { Traffic distribution } \\
\text { (vehicles /time interval) }\end{array}$ \\
\hline $\begin{array}{l}\mathbf{0 0 : 0 0 - 5 : 0 0} \\
\mathbf{2 2 : 0 0 - 2 3 : 0 0}\end{array}$ & 8 & $1-15$ \\
\hline $\mathbf{6 : 0 0 - 7 : 0 0}$ & 10 & $3-20$ \\
\hline $\begin{array}{l}\mathbf{8 : 0 0 - 9 : 0 0} \\
\mathbf{1 4 : 0 0 - 1 5 : 0 0} \\
\mathbf{2 0 : 0 0 - 2 1 : 0 0}\end{array}$ & 15 & $8-25$ \\
\hline $\begin{array}{l}\mathbf{1 0 : 0 0 - 1 1 : 0 0} \\
\mathbf{1 6 : 0 0 - 1 8 : 0 0}\end{array}$ & 25 & $16-40$ \\
\hline $\mathbf{1 2 : 0 0 - 1 3 : 0 0}$ & 18 & $10-28$ \\
\hline $\mathbf{1 9 : 0 0 - 2 0 : 0 0}$ & 20 & $14-30$ \\
\hline
\end{tabular}

\section{RESULTS AND DISCUSSIONS}

Simulations are performed through MATLAB codes. Traffic on lanes for each hour of the day is simulated in ten observation sets of 100 iterations each. The average value of these ten observation sets is considered and results are plotted. Performance analysis is done on the basis of percentage of vehicles left on a lane, number of times emergency vehicle is addressed and prioritized for clearance and on the basis of giving way to pedestrians with the maximum wait time to cross the lane. The obtained results are comparatively analyzed and discussed.

\section{A. Performance Analysis on the basis of Percentage of Vehicles Left on a Lane}

Simulated results of the three systems considered for performance analysis are shown in Fig. 13. The percentage of vehicles left on a single lane when the systems were simulated for ten sets of 100 iterations each at each hour of the day. The 
simulation result of vehicles left on a single lane is clearly represented in a graphical manner with $\mathrm{x}$-axis representing the time of day in hours, y-axis representing the PTCS, TDCS and designed FACS systems considered for comparison and $\mathrm{z}$-axis shows the percentage of vehicles left on the lanes. PTCS shows the worst performance with approximately $50 \%$ vehicles left on lanes in the peak traffic hours of the day. TDCS shows an improved performance with average of $25 \%$ vehicles left in the peak traffic hours. The designed FACS shows the best clearance with less than $5 \%$ vehicles left in the peak traffic hours and full clearance in the less and moderate traffic condition.

\section{B. Performance Analysis on basis of giving Highest Priority to Emergency Transit}

Another important criterion to analyze the system performance is the number of times highest priority is given to the lane on which emergency transit is detected or in other words we can say that the emergency transit is addressed as soon as it is detected on a particular lane. Emergency vehicle is assumed to arrive at any lane of the intersection randomly with a certain probability. The simulation results obtained are shown with the help of a bar graph in Fig. 14. The designed FACS shows $100 \%$ performance in addressing the Emergency vehicle at all traffic flow conditions throughout the day irrespective of traffic flow condition. The simulation result obtained for PTCS and TDCS clearly signifies that they are not able to address emergency transit effectively.

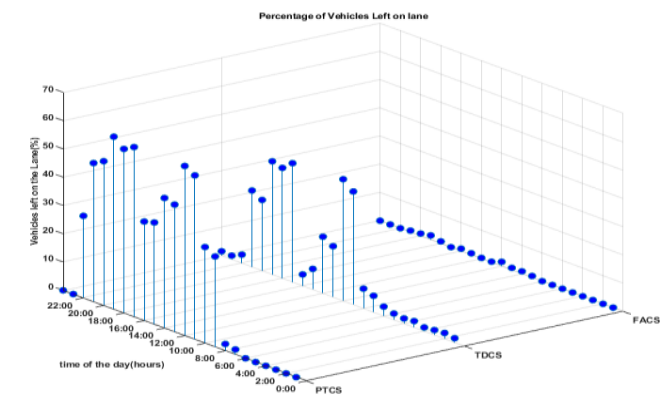

Fig. 13. Percentage of Vehicles Left on Lanes.

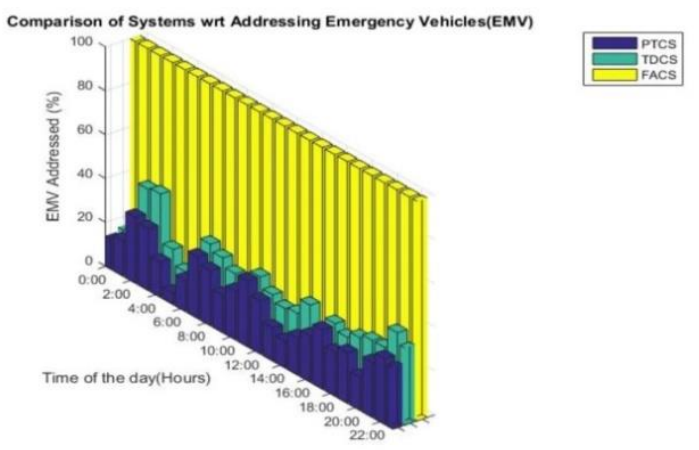

Fig. 14. Comparison of Percentage of Times Emergency Transit is addressed.

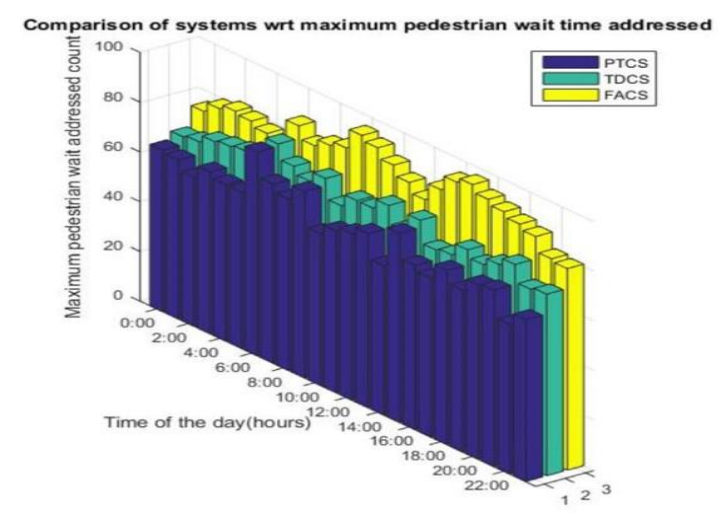

Fig. 15. Comparison of Percentage of Times Maximum Pedestrian Wait Time is addressed.

\section{Performance Analysis on the basis of giving way to Pedestrians}

To evaluate the performance of the designed system with respect to assisting pedestrians waiting at the intersection, the PTCS, TDCS and FACS systems are simulated in ten sets of 100 iterations for each hour of the day. Fig. 15 illustrates the measure of number of times the lane with pedestrians waiting for maximum time is given highest priority for red light to allow pedestrians to cross. The data plotted for each hour of the day is an average of the values obtained in ten observation sets of 100 iterations each. The designed FACS shows maximum clearance of pedestrians with highest waiting time and the performance of the system is better even in the peak traffic hours.

\section{CONCLUSION}

The proposed FACS performs better than the PTCS and TDCS due to its flexible design. The simulation results clearly show that the designed system gives better clearance to vehicles, emergency transit and also to pedestrians in all traffic flow conditions throughout the day. This system can be realized and implemented on any major traffic intersection to address all types of movements and enhance the traffic as well as pedestrian handling capability of the intersection.

In future these smart intersections can be interconnected through the major routes of the city such as routes leading to hospitals to give a thorough passage to emergency transit.

\section{ACKNOWLEDGMENT}

The authors are grateful to SHUATS, Prayagraj (formerly Allahabad) for providing the opportunity to write this research paper.

\section{REFERENCES}

[1] Ali MAH, Mailah M, Jabbar WA, Moiduddin K, Ameen W, Alkhalefah H. Autonomous Road Roundabout Detection and Navigation System for Smart Vehicles and Cities Using Laser Simulator-Fuzzy Logic Algorithms and Sensor Fusion. Sensors. 2020; 20(13):3694.

[2] Agrawal A, Paulus R. Intelligent traffic light design and control in smart cities: a survey on techniques and methodologies. International Journal of Vehicle Information and Communication Systems. 2020;5(4):436-81. 
[3] Guerrero-Ibáñez J, Zeadally S, Contreras-Castillo J .Sensor technologies for intelligent transportation systems. Sensors 2018;18(4):1212.

[4] Zadeh, Lotfi A. "On fuzzy algorithms." In fuzzy sets, fuzzy logic, and fuzzy systems: selected papers By LotfiA Zadeh, pp. 127-147. 1996.

[5] Koukol M, Zajíčková L, Marek L, Tuček P. Fuzzy logic in traffic engineering: a review on signal control. Mathematical Problems in Engineering. 2015 Jan 1;2015.

[6] Rahman SM, Ratrout NT. Review of the fuzzy logic based approach in traffic signal control: prospects in Saudi Arabia. Journal of transportation Systems engineering and information Technology. 2009 Oct 1;9(5):58-70.

[7] Abdel Nasser H. Zaied, Woroud Al Othman "Development of a fuzzy logic traffic system for isolated signalized intersections in the State of Kuwait", Expert Systems with Applications: An International Journal, Volume 38 Issue 8, 9434-9441, August, 2011.

[8] Alam J, Pandey MK, 'Development of Intelligent Traffic Light System Based On Congestion Estimation Using Fuzzy Logic'IOSR Journal of Computer Engineering (IOSR-JCE) e-ISSN: 2278-0661, p- ISSN: 22788727 Volume 16, Issue 3, Ver. VI ,May-Jun. 2014, pp 36-44.

[9] Ge, Yan. "A two-stage fuzzy logic control method of traffic signal based on traffic urgency degree." Modelling and Simulation in Engineering 2014.

[10] Alam J, Pandey MK. Design and analysis of a two stage traffic light system using fuzzy logic. J. Inf. Technol. Softw. Eng. 2015;5(03).

[11] Rocha, J., Martínez, S., Menchaca, J., Villanueva, J., Berrones, M., Cobos, J. and Agundis, D. 'Fuzzy Rules to Improve Traffic Light Decisions in Urban Roads', Journal of Intelligent Learning Systems and Applications, Vol. 10, pp. 36-45, 2018.

[12] Vogel, Alan, IzidorOremović, Robert Šimić, and Edouard Ivanjko. "Fuzzy Traffic Light Control Based on Phase Urgency." In 2019 International Symposium ELMAR, pp. 9-14. IEEE, 2019.

[13] M. Firdous, F. U. Din Iqbal, N. Ghafoor, N. K. Qureshi and N. Naseer, "Traffic Light Control System for Four-Way Intersection and T-
Crossing Using Fuzzy Logic," 2019 IEEE International Conference on Artificial Intelligence and Computer Applications (ICAICA), Dalian, China, 2019, pp. 178-182.

[14] Onoriode UM, Guo T, Pan S. Division of Green Time for Adaptive Traffic Signal Control Method based on Fuzzy Logic. International Journal of Transportation Systems. 2019;4.

[15] Jha, Mohit, and Shailja Shukla. "Design Of Fuzzy Logic Traffic Controller For Isolated Intersections With Emergency Vehicle Priority System Using MATLAB Simulation." arXiv preprint arXiv:1405.0936 (2014).

[16] Homaei, Hamed, S. R. Hejazi, and Seyed Ali Mohamad Dehghan. "A new traffic light controller using fuzzy logic for a full single junction involving emergency vehicle preemption." Journal of Uncertain Systems 9, no. 1 (2015): 49-61.

[17] Shelke, M., Malhotra, A., \& Mahalle, P. N. "Fuzzy priority based intelligent traffic congestion control and emergency vehicle management using congestion-aware routing algorithm" Journal of Ambient Intelligence and Humanized Computing (2019) doi:10.1007/s12652-019-01523-8.

[18] W. K. Saad, Y. Hashim and W. A. Jabbar, "Design and Implementation of Portable Smart Wireless Pedestrian Crossing Control System," in IEEE Access, vol. 8, pp. 106109-106120, 2020, doi: 10.1109/ACCESS.2020.3000014.

[19] Pau, Giovanni, TizianaCampisi, Antonino Canale, Alessandro Severino, Mario Collotta, and Giovanni Tesoriere. "Smart pedestrian crossing management at traffic light junctions through a fuzzy-based approach." Future Internet 10, no. 2 (2018): 15.

[20] Aria, Muhammad "New fuzzy logic system for controlling multiple traffic intersections with dynamic phase selection and pedestrian crossing signal." J. Eng. Sci. Technol 14 (2019): 1974-1983.

[21] Gerlough, Daniel L., and Andre Schuhl. "Use of Poisson Distribution in Highway Traffic. The Probability Theory Applied to Distribution of Vehicles on Two-Lane Highways." (1955). 